\title{
PERFORMANCE EVALUATION OF ROUTING PROTOCOLS FOR DELAY TOLERANT NETWORKS
}

\author{
Luming Wan ${ }^{1}$, Feiyang Liu ${ }^{1}$, Juan Zhang $^{2}$, and Haibo Zhang ${ }^{1}$ \\ ${ }^{1}$ Department of Computer Science, University of Otago, Dunedin, New Zealand \\ \{lwan, feiyang, haibo\}@cs.otago.ac.nz \\ ${ }^{2}$ School of Reliability and System Engineering, \\ Beihang University, Beijing, China \\ juanzhang@buaa. edu. cn
}

\begin{abstract}
.
Delay Tolerant Network (DTN) is a promising technology which aims to provide efficient communication between devices in a network with no guaranteed continuous connectivity. Most of the existing routing schemes for DTNs achieve message delivery through message replication and forwarding. However, due to the lack of contemporaneous end-to-end communication path, designing routing protocols that can achieve high delivery rate with low communication overhead is a challenging problem. Some routing protocols appear with high similarity, but their performance are significantly different. In this paper, we evaluate several popular routing protocols in DTNs, including Epidemic, Spray and Wait, PRoPHET, and 3R through extensive trace-driven simulations. The objective is to evaluate the performance of different routing schemes using different data traces and investigate the optimal configuration setting for each routing scheme. This paper provides important guidances on the design and selection of routing protocols for given delay tolerant networks.
\end{abstract}

\section{KEYWORDS}

Delay Tolerant Network, Performance Evaluation, Routing

\section{INTRODUCTION}

The increasing popularity of ubiquitous computing and communication leads to a huge desire on data exchanging between wireless mobile devices, e.g. cell phones, laptops, tablets, and other carriable devices, regardless of whether any guaranteed end-to-end connection exists. Delay tolerant networks (DTNs) address the technical issue on communication between devices that lose continuous connectivity due to mobility. Currently, DTNs have been applied to a vast of areas, including vehicular networks[1], wildlife tracking[2], and social network analysis[3], etc. However, DTNs are still appearing with numerous of limitations. For example, due to the lack of synchronous end-to-end connectivity in DTNs, mobile devices have to carry the messages and forward them opportunistically upon encountering the destinations, or forward messages to other relays to help the delivery. This could result in incredible long transmission delay and high error

David C. Wyld et al. (Eds) : WiMo, ITCSE, ICAIT, NC - 2015

pp. 01-12, 2015. C CS \& IT-CSCP 2015

DOI : 10.5121/csit.2015.51001 
rate. In addition, the constraints of wireless devices, such as storage capacity, communication bandwidth and battery power, can significantly impact the delivery rate. Hence, routing protocols for DTNs should be able to adapt to the network variation and efficient to make use of the hardware resources.

Recent studies exhibit that most of the existing routing protocols designed for DTNs appear with high similarity in concepts, but their performance are significantly disparate. Epidemic[4] performs robust and blind flooding of messages to the network. Spray and Wait[5] is a floodingcontrolled version of Epidemic, as it simply limits the amount of messages that can be flooded to the network. In most prediction-based schemes, such as PRoPHET[6], MaxProp[7] and PER[8], the message forwarding decisions are made based on a quality metric called encounter predictability which is accumulated from each time of encounter. However, the above predictionbased schemes ignore some important encounter information, such as the contact time. Some studies demonstrate that human activities appear with high repetition, such as weekly meetings. The performance of routing schemes could be enhanced significantly through the exploitation and utilization of those regular patterns. 3R[9] is a fine-grained history-based routing scheme, by which the contact time of encounters are perfectly recorded. Due to the maintenance of finegrained encounter history, the encounter predictability in $3 \mathrm{R}$ is time-dependent, that is, it can be calculated based on only the past contacts that occurred in the same period as the lifetime of the packet, rather than making a long-term average estimation as in most prediction-based protocols, such as PRoPHET. In addition, 3R is a forwarding-based scheme, each node always forwards the original message to the next good relay rather than transmitting replicas as in all the protocols above. However, single-copy for each message is not ideal for DTNs since message could be easily lost due to unstable connectivity and other uncertainties.

It is difficult to design an absolutely perfect routing protocol for all DTN applications. The performance of a routing protocol can be affected by a large number of factors, such as the popularity and active rate of nodes, different setting of parameters for mobile devices, etc. In this paper, we evaluate the performance of several most popular routing protocols, including Epidemic, Spray and Wait, PRoPHET, and 3R through trace-driven simulations. Also, we investigate the impact of different parameters of Spray and Wait and PRoPHET to achieve their optimal settings. The contribution of this paper is to provide some important guidances on routing protocol design and selection for delay tolerant networks.

The reminder of this paper is organized as follows: Section 2 introduces the protocols we are going to evaluate in detail. Section 3 introduces the data traces and experiment setup. Section 4 shows the performance evaluation in two perspectives. Firstly, we present the impact of different parameter settings for Spray and Wait, and PRoPHET protocols. Then, we evaluate the performance of all the protocols with their optimal parameter settings. The paper is concluded in Section 5 .

\section{ROUTING PROTOCOLS}

This section introduces four protocols of our comparisons in detail, which are Epidemic, PRoPHET, Spray and Wait, and 3R respectively. 


\subsection{Epidemic}

Epidemic[4] is a pure flooding-based routing protocol for DTNs. Each device (source or relaying node) always propagates message replicas to all of the contactable nodes until the message is received by the destination node or the message deadline expires. Due to the nature of unlimited flooding, Epidemic is able to achieve the best message delivery rate when each node has an infinite memory buffer, but its performance deteriorates significantly when each node only has limited resources. This is because numerous of message replicas have to be dumped due to the memory overflow.

\subsection{Spray and Wait}

Similar to Epidemic, Spray and Wait [5] is also a flooding-based routing protocol, but it controls the message flooding by directly limiting the number of replicas that an original message can produce. Each message can only propagate a constant amount of replicas in the spray phase. After that, the original message and all of its replicas switch to the wait phase, that is, those messages only wait for the destination node encountering their holders rather than further spreading replicas to other relays. Binary Spray and Wait is a derivation of original version, as nodes spread the replicas of a message in a binary tree manner. Suppose a message is allowed to spread $L$ replicas, the source node will generate $L$ replicas in the beginning and spread them among encountered relays. Suppose any node $A$ that has $n(1 \leq n \leq L)$ replicas of message $m$, and it encounters a node $B$ with no replica of $\$ \mathrm{~m} \$$ previously. $A$ will send $\left\lceil\frac{n}{2}\right\rceil$ replicas to $B$ and keep the rest of $\left\lfloor\frac{n}{2}\right\rfloor$ replicas to wait for more relaying nodes. When only one replica is left in $A$ or any other relaying node, they stop spreading any replicas and wait for encountering the destination node. Spray and Wait can achieve a trade-off between the delivery rate and transmission overhead by setting a proper limitation of $L$.

\subsection{PRoPHET}

PRoPHET[10] is a prediction-based scheme and it is one of the few DTN routing protocols that have an IETF draft. It implements a quality metric called encounter predictability to measure the capability of the encountering nodes whether or not it can transmit the message to the destination. A replica of a message is propagated to the encountering node if it has a higher predictability than its holder. This guarantees that each time a message can always be propagated to a better relay. The encounter predictability is integrated in three perspectives, which are direct probability, transitivity and ageing. Direct probability is the probability of transmitting a message directly between two nodes. It is updated whenever two nodes directly encounter each other as follows:

$P(a, b)=P_{\text {old }}(a, b)+\left(1-\delta-P_{\text {old }}(a, b)\right) \times P_{\text {encounter }}$,

where $P_{\text {old }}(a, b)$ is the encounter probability of node $a$ and $b$ before the current encounter occurs,

$P_{\text {encounter }} \in[0,1]$ is a scaling factor at which the probability increases on encounters, and $\delta$ is a small positive value to set an upper bound on $P(a, b)$.

Transitivity estimates the probability of indirect contact that through multiple-hop relays, as shown below: 
$P(a, c)=P_{\text {old }}(a, c)+\left(1-P_{\text {old }}(a, c)\right) \times P(a, b) \times P(b, c) \times \beta$,

Where $P(a, c)$ is the encounter probability between node $a$ and $c$ through relay node $b, P_{\text {old }}(a, c)$ is the probability from previous time of update, and $\beta \in[0,1]$ is a scaling factor that decides how large the impact of transitivity should have on the encounter predictability.

PRoPHET uses an ageing mechanism to decay the encounter predictability and eliminate the long-time inactive nodes, as shown below:

$P(a, b)=P_{\text {old }}(a, b) \times \gamma^{k}$

where $\gamma \in[0,1]$ is the ageing constant, and $K$ is the number of time units elapsed since the last time $P(a, b)$ was aged.

As shown in the above Equations, there are three important parameters for PRoPHET protocol: scaling factors for direct delivery $P_{\text {encounter }}$ and transitivity $\beta$, and the ageing constant $\gamma$. They have significant influence on the performance and overheads of Prophet scheme. PRoPHET can be an efficient routing protocol by setting the proper parameters if the encountering patterns in DTNs are predictable, because the messages and replicas are only forwarded to the relays with more benefits on message delivering. At present, there are two versions of PRoPHET protocol: PRoPHET'03[6] and PRoPHET'12[10]. They have different recommendation settings for these parameters, while the principles of message forwarding for both versions are basically identical.

\subsection{R}

3R[9] characterizes the encounter history in a fine-grained form by storing more information for each encounter, including the nodes that the encounter occurs among, the start time and the end time of this encounter. Each node maintains a table for the encounter information of its contacted nodes. The fine-grained history in a node will group the contacts according to different types of the day \{weekday, weekend\}. Each item in the table represents a fixed time interval of the real world (e.g. 1 hour), and it records the overall contact frequency between the table holder and the specified node at exact the same period in the past. Suppose a message $m$ has a lifetime that spans $k$ slots. Let $F_{i}^{a}$ be the number of contacts of node $a$ that occurred at a time slot $i$ in the past, and $F_{a b}^{i}$ be the number of contacts occurred between node $a$ and $b$ at time slot $i$, the estimated contact probability $\mathrm{p}_{\mathrm{ab}}^{\mathrm{i}}$ at slot $i$ is:

$p_{a b}^{i}=\frac{F_{a b}^{i}}{F_{a}^{i}}$

Suppose the lifetime of a message $m$ spans $k$ time slots, the overall probability that message $m$ can be delivered from node $a$ to node $b$ before it expired is estimated by integrating the probabilities of these $k$ slots as follows:

$P_{a b}(k)=1-\prod_{i=0}^{k}\left(1-p_{a b}^{i}\right)$.

Due to the maintenance of fine-grained history, a time-dependent forwarding prediction is enabled by only figuring out the delivery probability within the lifetime of a message, rather than calculating a long-term average estimation. Similar to PRoPHET, 3R also compares the 
predictability and forwards messages to nodes with higher delivery probability. Whereas, $3 \mathrm{R}$ only directly forwards the original message to the next relay, instead of sending a replica as in PRoPHET. Due to the predictability calculation based on fine-grained contact history and singlecopy message forwarding, $3 \mathrm{R}$ can effectively reduce delivery overheads with no much loss on message delivery rate.

\section{DATA TRACES AND EXPERIMENT SETUP}

\subsection{Data Traces}

We evaluate above four representative routing protocols for DTNs with extensive trace-driven simulations. To increase the accuracy and reliability of our evaluation, we use two realistic DTN data traces: INFOCOM[11] and MIT Reality[12], which are obtained from the open-source website, CRAWDAD[13].

- INFOCOM: This data trace consists of Bluetooth sightings and it records 4 days of contacts occurred during the INFOCOM'05 conference. In the experiment, 24 internal devices monitored the network and more than 200 external devices were discovered. Each device had a scan granularity of 120 seconds, and each scan lasted for 5 seconds.

- MIT Reality: This trace contains the contact information including communication, proximity, and location from faculties and students at MIT over the course of the 20042005 academic year. To collect this trace, 89 devices recorded the contacts, and more than 20,000 devices were discovered. Each device scanned to discover neighbors every 5 minutes. In our simulations, we use the devicespansubtrace that records Bluetooth contacts for 1 month.

\subsection{Experiment Setup}

In our simulations, the routing schemes are evaluated using the same message trace and contact trace. To avoid the negligible delivery rate caused by numerous long-time inactive nodes which actually seldom communicate, we randomly select the source node and the destination node of a message from 20 most active nodes during the lifetime of this message. In the INFOCOM trace, each source generates a message with the probability of 0.15 in every 600 seconds, whereas the probability is 0.6 in the MIT Reality trace. The lifetime of each message varies from 2 hours to 3 days if not specially notified. The size of each generated message is randomized from $2 \mathrm{k}$ bytes to $100 \mathrm{k}$ bytes.

For the MIT Reality trace, the network is warmed up for one week at the beginning of each simulation run. This avoids the inaccurate prediction on encounter probability for predictionbased protocols, i.e.PRoPHET and 3R, when the network is just booted up. Also, we reserve 3 days at the end of simulations to avoid any message left in the network after the simulation is completed. During these warm-up and sinking periods, messages are not allowed to be generated. This process is not available for the INFOCOM data trace, since it only contains 4 days of contact traces. 


\section{PERformance Evaluation}

In this section, we firstly exhibit the performance of Spray and Wait and PRoPHET with different parameter settings using the INFOCOM data trace, since they have some specific parameters. Then we investigate the performance of all four protocols, i.e. Epidemic, Spray and Wait, PRoPHET, and 3R, through trace-driven simulations. The performances of these four protocols are investigated in two perspectives: different memory buffer sizes and packet lifetimes. The performance is evaluated using the following three metrics:

- average message delivery rate: the proportion between the number of successfully delivered messages against the number of original messages.

- message overhead $(M O)$ : the ratio of the number of replicas against the number of original messages.

- communication overhead $(\mathrm{CO})$ : the ratio of the difference between the number of delivered messages and hop-by-hop transmissions to the number of delivered messages.

The parameter setting for Spray and Wait is slightly different in each data trace in accordance with the characteristic of the data trace. The Binary Spray and Wait protocol is implemented in the entire experiments as it performs slightly better than its original version. Due to the huge difference on popularity of nodes in these two traces, the maximum number of replicas for Binary Spray and Wait with INFOCOM trace is set to 5, and 70 with MIT Reality trace. The parameters in PRoPHET are configured with the values provided in PRoPHET'12[10] by default. All the nodes are set with infinite memory buffer if not specified. All the protocols dump messages using the First In First Out policy when the message buffer is full, that is, the first message in the buffer queue (oldest) is dumped to prevent buffer overflow.

\subsection{Impact of Parameters for Spray-and-Wait and PRoPHET}

Spray-and-Wait: Fig. 1 shows the performance of the original and binary version of Spray and Wait routing protocol with different maximum number of replicas. In Fig.1(a), it can be seen that the original Spray and Wait achieves higher delivery rate than the binary version when the maximum number of replicas is less than 10 . With the further increase on the number of replicas, the binary version performs better than the original version. That is because the original scheme can only spread the replicas around the neighbours, while the binary scheme can spread the replicas more widely in a binary-tree manner if enough number of replicas are allowed. We can seethe delivery rate of both protocols remain steady when the maximum replicas exceeds 40 .

The highest average delivery rate achieved by the original and the binary versions are 0.9314 and 0.9382 , respectively. That means the number of replicas are enough for two Spray and Wait protocols with no further benefits for more replicas. Meanwhile, MO and $\mathrm{CO}$ for both schemes appear with the similar increasing trend as shown in Fig. 1(b) and (c). The MO and CO of the original Spray and Wait increase apparently with the maximum replicas less than 40, and they stay constant afterwards also due to the limitation on the number of neighbours, whereas both two kinds of overheads of Binary Spray and Wait grow almost linearly since the replicas can be spread to more relay nodes on the network. From these figures, it can be seen that with a large number of replicas, the multi-hop routing of Binary Spray and Wait is able to perform better than the only two-hop routing in original version, but it suffers almost twice of overheads than the original version. 


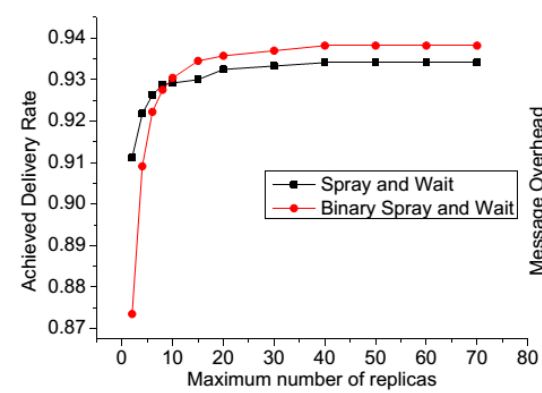

(a)

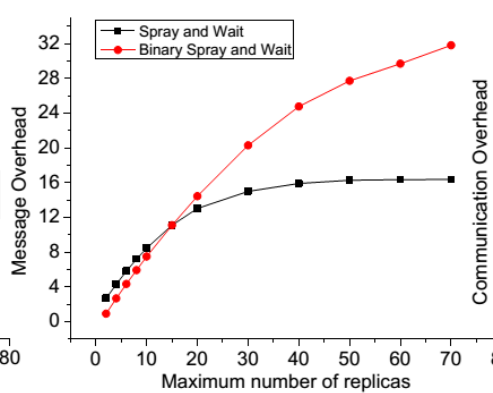

(b)

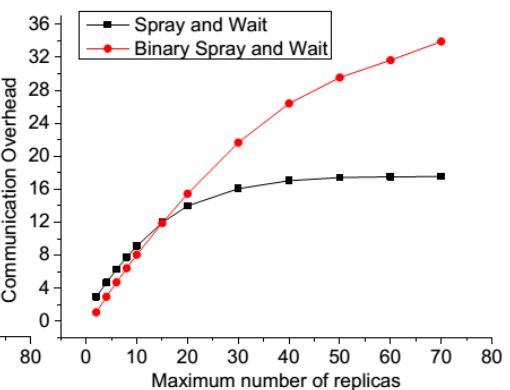

(c)

Fig. 1. The performance of Spray and Wait with different number of message replicas, (a) delivery rate, (b) message overhead, (c) communication overhead.

The highest average delivery rate achieved by the original and the binary versions are 0.9314 and 0.9382 , respectively. That means the number of replicas are enough for two Spray and Wait protocols with no further benefits for more replicas. Meanwhile, MO and CO for both schemes appear with the similar increasing trend as shown in Fig. 1(b) and (c). The MO and CO of the original Spray and Wait increase apparently with the maximum replicas less than 40, and they stay constant afterwards also due to the limitation on the number of neighbours, whereas both two kinds of overheads of Binary Spray and Wait grow almost linearly since the replicas can be spread to more relay nodes on the network. From these figures, it can be seen that with a large number of replicas, the multi-hop routing of Binary Spray and Wait is able to perform better than the only two-hop routing in original version, but it suffers almost twice of overheads than the original version.

PRoPHET: The performance of PRoPHET routing protocol is critically impacted by the parameters shown in Table 1. PRoPHET provides totally different recommended parameters in their 2012 version [10] compared to the 2003 version [6].

Table 1.PROPHET recommended parameters

\begin{tabular}{|c||c|c|c|}
\hline Parameters & $\mathrm{P}_{\text {encounter }}$ & $\beta$ & $\gamma$ \\
\hline \hline PRoPHET 2003 & 0.75 & 0.25 & 0.98 \\
\hline PRoPHET 2010 & 0.5 & 0.9 & 0.999 \\
\hline
\end{tabular}

Fig. 2 shows the performance of PRoPHET'03 and PROPHET'12. In these experiments, each node has infinite memory buffer to store messages and replicas. The principles of message forwarding for these two versions are basically identical. It can be seen that in Fig. 2(a), the delivery rate is increased by $0.5 \%$ with the parameters of PRoPHET'12 in comparison with PRoPHET'03. A larger ageing parameter $\gamma$ makes PROPHET'12 better to tolerate the variation of contact patterns and achieve higher delivery rate. However, both $\mathrm{MO}$ and $\mathrm{CO}$ are raised by $6.1 \%$ and 5.4\% respectively as shown in Fig. 2(b). This is because a larger $\beta$ in PRoPHET'12 leads to a higher impact on the transitive connectivity, and a lower $\mathrm{P}_{\text {encounter }}$ reduces the impact of direct delivery. Hence, more replicas need to be generated and forwarded by the relay nodes. 


\subsection{Performance Evaluation with Different Memory Sizes}

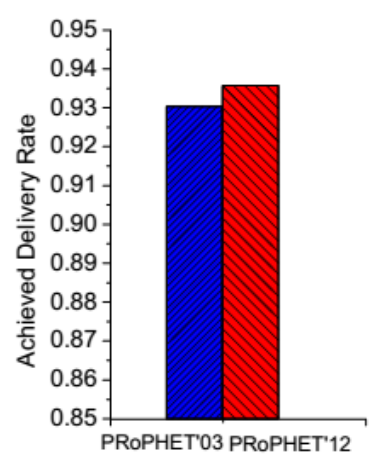

(a)

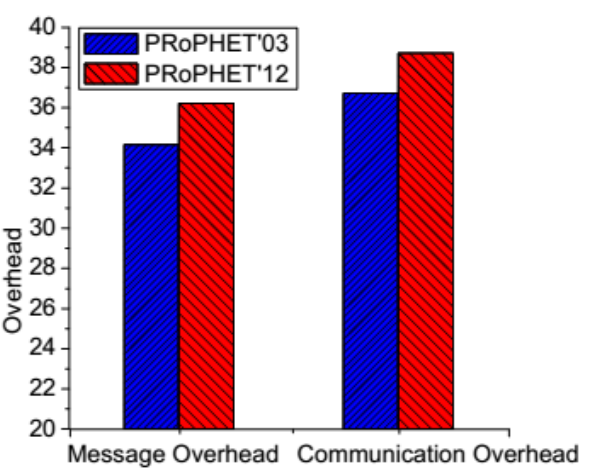

(b)

Fig.2.PRoPHET'03 versus PRoPHET'12, (a) delivery rate, (b) overheads.

In each mobile device, the memory buffer used for message delivery always has a limited size. The available memory has huge influence on the delivery rate and overheads. We evaluate the performance of four routing protocols with different memory sizes using INFOCOM and MIT Reality traces.

(1) INFOCOM Trace: In Fig. 3(a), we evaluate the influence of memory size on the delivery rate for different routings. It can be seen that the delivery rate of all four routing protocols have a sharp increasing trend with a small memory buffer $(<10 \mathrm{MB})$, and then they tend to remain stable even if the memory buffer size keeps growing. Binary Spray and Wait has more outstanding performance than other three routings when each node has a memory buffer of $80 \mathrm{M}$ bytes or less. It reaches its maximum delivery rate of 0.923 with the memory buffer size increasing to $60 \mathrm{M}$ bytes, and the delivery rate tends to be stable afterwards. That is because it is a controlled flooding scheme (5 replicas) and has less message dropped due to memory overflow than other routings. When the memory size exceeds $60 \mathrm{MB}$, there will be no message dumps for this routing and the delivery rate will be stable. Similarly, 3R also has better performance with a smaller memory buffer size compared with Epidemic and PRoPHET. It has less requirement on memory size because of single-message and its limitation on message forwarding. However, it reaches the maximum delivery rate of 0.792 at the memory size of $40 \mathrm{M}$ bytes, which is much smaller compared with the other three protocols. That is because $3 \mathrm{R}$ only forwards the original message. Although the delivery rates of Epidemic and PRoPHET raise slowly, their maximum delivery rates are higher than $3 R$ and Spray and Wait (0.941 and 0.930 respectively) since they are memory-hungry schemes. Epidemic protocol needs large amounts of memory for unlimited flooding and can achieve the highest delivery rate with enough memory. PRoPHET also needs a large memory size for transmitting the amounts of messages and replicas.

Fig. 3(b) and (c) evaluate the overheads with different memory sizes. We can see that Epidemic always has the highest $\mathrm{MO}$ and $\mathrm{CO}$ due to the nature of unlimited flooding. Since it needs to generate more replicas when encountering relay nodes with high predictability, PRoPHET also has higher overheads, which is almost half of the overheads as Epidemic. Because the maximum number of replicas in Binary Spray and Wait is set to 5, the maximum MO and CO of Binary Spray and Wait scheme tend to 5 even with a much larger memory size. Since $3 \mathrm{R}$ is a singlemessage forwarding-only scheme, there is no message overhead. The $\mathrm{CO}$ of $3 \mathrm{R}$ is also lowest because the message is only forwarded to a node with higher predictability according to fine- 
grained encounter history. The CO of Binary Spray and Wait and 3R appear with a decreasing trend as the memory size increases, since they have limitations on message forwarding and a larger memory size increases the number of delivered messages by reducing message dumps.
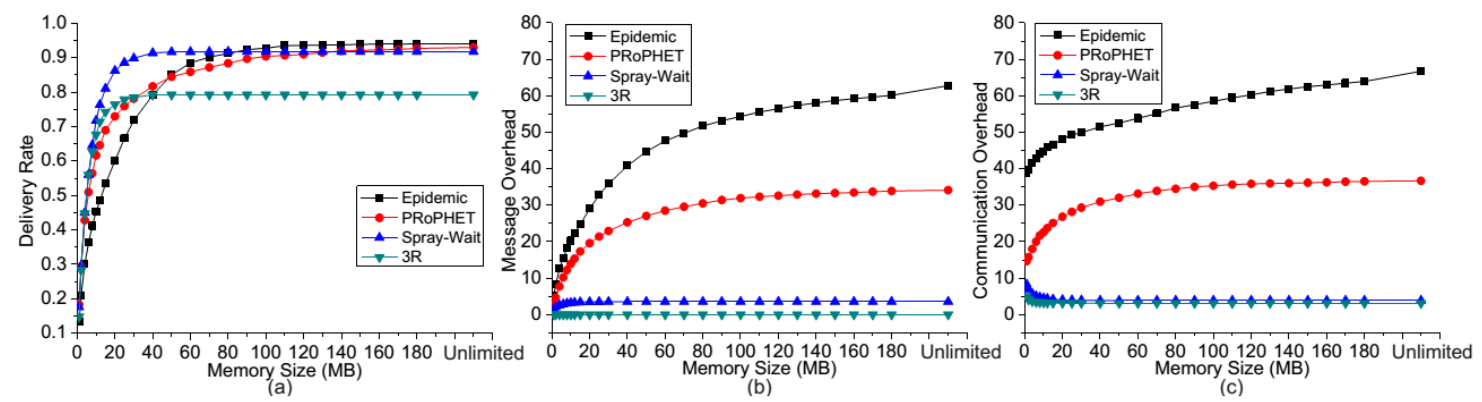

Fig.3.Performance evaluation with different memory sizes using INFOCOM trace,(a) delivery rate, (b) message overhead, (c) communication overhead.
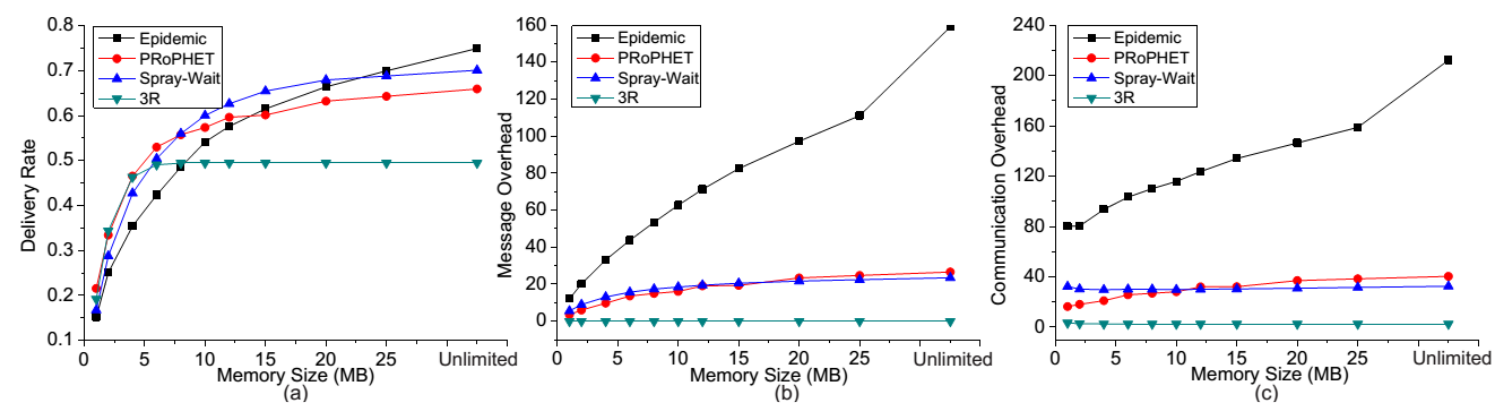

Fig. 4.Performance evaluation with different memory sizes using MIT Reality trace, (a) delivery rate, (b) message overhead, (c) communication overhead.

(2)MIT Reality Trace: As shown in Fig. 4(a), the delivery rate of each routing protocol has similar trend as the memory size increases. However, PRoPHET and 3R have better performance than Epidemic and Spray and Wait when the memory size is small $(<5 \mathrm{MB})$. Since the MIT Reality trace lasts for 1 month and these two routings can make use of the contact history to get more accurate prediction for message forwarding, a smaller memory is relatively enough due to less replicas and message forwarding. As the memory size increases, Epidemic and Spray and Wait achieve higher delivery rate because a large number of flooding replicas can be tolerated in the memory with few of message dumps.

Similarly, the message overhead (MO) in Fig. 4(b) and communication overhead in Fig. 4(c) vary in the same way as the memory size increases. Since there are more nodes in the MIT Reality data trace than in INFOCOM trace, the overheads of Epidemic are significantly increased. That means Epidemic is not suitable for DTNs with a large number of devices. We can see that the overheads of Spray and Wait increase with the increase of maximum allowed replicas (from 5 in INFOCOM trace to 70 in MIT Reality trace). PRoPHET and 3R protocols have lower overhead for both data traces, which is determined by the popularity of nodes and the activeness of each node. 
From this set of simulations, it can be seen that flooding-based routing protocols can achieve better delivery rate, but require much larger message memory. Whereas, the prediction-based routing protocols are more memory efficient, and can achieve desirable delivery rate with very low overheads as they have an accurate prediction on the contact patterns.

\subsection{Performance Evaluation with Different Packet Lifetimes}

Each message may experience an unpredictable delivery latency in DTNs due to the lack of guaranteed continuous end-to-end connectivity. Therefore, in order to achieve more effective communication for DTNs, each message needs a suitable lifetime either by users setting or default configuration of specific devices.In this set of simulations, we evaluate the influence of different packet lifetimes for the routing algorithms using INFOCOM and MIT Reality data traces respectively. We assume each node has an infinite buffer size.

(1) INFOCOM Trace:Fig. 5 shows the delivery rate and overheads of four routing protocols with different setting of packet lifetimes using the INFOCOM trace. In Fig. 5(a), it can be seen that for each routing protocol the delivery rate appears with a dramatic increase when the packet lifetime increases from 6 to 30 hours. For example, the delivery rate increases from 0.454 to 0.832 for $3 \mathrm{R}$ routing. The reason is that the probability of directly encountering the destination or the message delivered to the destination by multi-hop forwarding grows when the packet lifetime increases. This increasing trend slows down when the packet lifetime exceeds 30 hours. That is because the data trace only lasts 4 days, and the contact pattern is short-time dependent. The delivery rate will not increase even with a large packet lifetime since the destination node may only be active in a short time period. The overheads shown in Fig. 5(b) and (c) also demonstrate that, for Epidemic and PRoPHET, the overheads increase as the packet lifetime increases, since more replicas can be generated and forwarded to the destination. Whereas, the lifetime is enough long for message delivery when it exceeds 30 hours in this trace. The overheads of Spray and Wait and 3R keep stable with different packet lifetime, because the number of replicas is limited in Spray and Wait and 3R is a single-message forwarding based scheme.

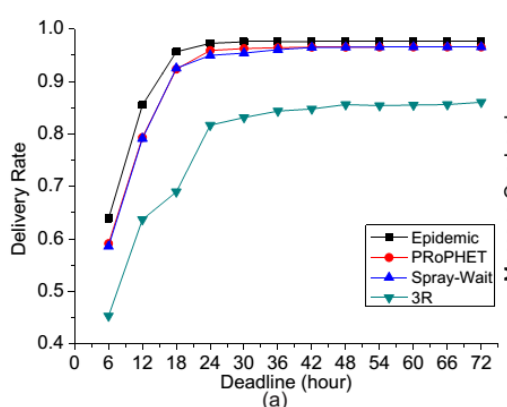

(a)

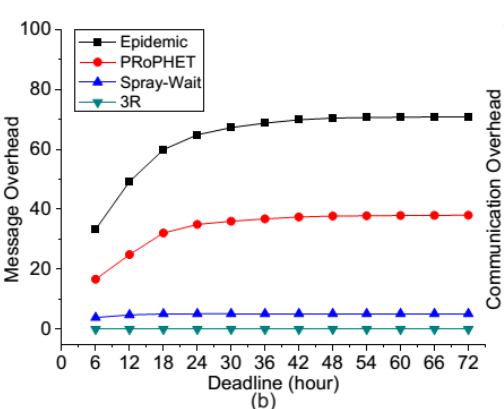

(b)

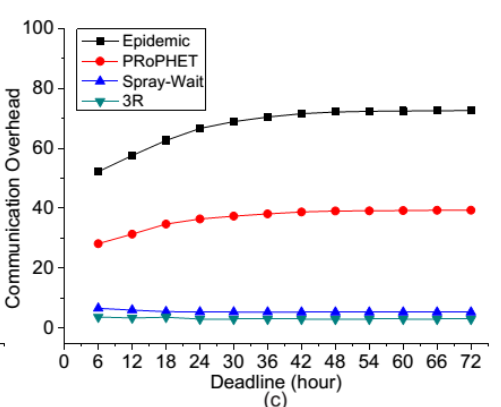

(c)

Fig.5.Performance evaluation with different packet lifetimes using INFOCOM trace, (a) delivery rate, (b) message overhead, (c) communication overhead. 

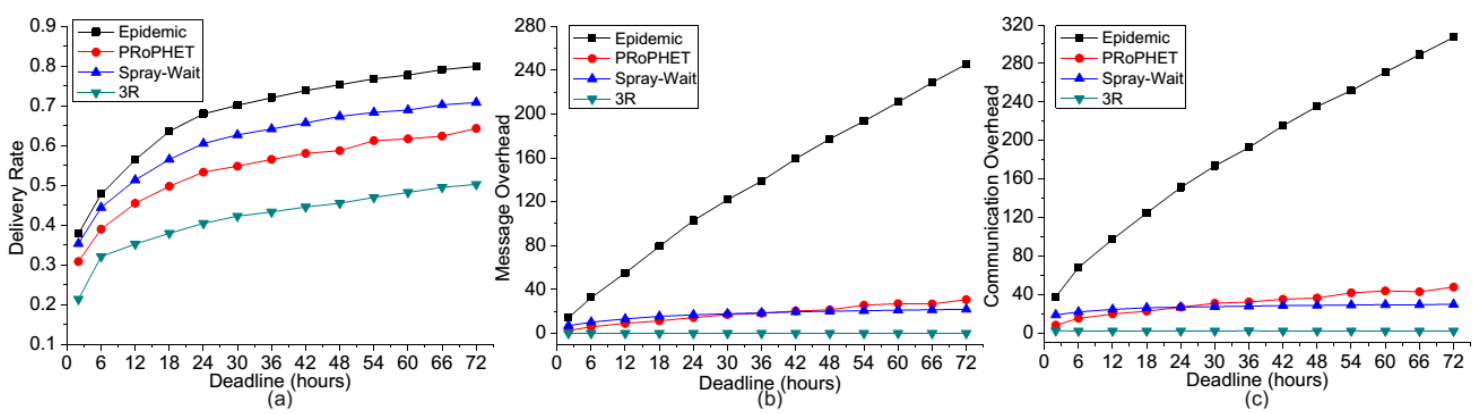

Fig.6.Performance evaluation with different packet lifetimes using MIT Reality trace, (a) delivery rate, (b) message overhead, (c) communication overhead.

(2) MIT Reality Trace: The simulation results with MIT Reality trace are similar as shown in Fig. 6. It can be seen in Fig.6(a) that the delivery rate for all the routing protocols keep growing as the packet lifetime increases. It is because MIT Reality trace lasts for 1 month and it is longtime dependent, thus a longer packet lifetime always provides a larger probability of successful delivery. In this simulation, as shown in Fig.6(b) and (c) the overheads of Epidemic increase dramatically both due to longer packet lifetime and larger device population. Whereas, the overheads variations for other routing protocols are similar to the results using INFOCOM data trace.

This set of simulations demonstrate that a longer packet lifetime can provide a higher delivery rate for DTNs, since there is more chance to encounter the destination node either by direct contact or multi-hop message forwarding. The overheads of flooding-based routings increase significantly, while the prediction-based routings are more efficient, especially when the DTNs have long-time dependent contact patterns.

\section{CONCLUSION}

Due to the property of Delay Tolerant Networks for no guaranteed continuous connectivity, choosing a proper routing protocol and finding its optimal configuration are challenging problems. In this paper, we evaluate some existing representative routing protocols for DTNs through extensive trace-based simulations. Flooding-based routing protocols, such as Epidemic and Spray and Wait, can achieve better delivery rate by sacrificing the memory, while predictionbased routings, like PRoPHET and 3R, are more efficient when considering the delivery overheads. This paper provides important guidances for routing protocols design. Our future work is to design an efficient routing protocol for DTNs with high delivery rate and low delivery overheads.

\section{REFERENCES}

[1] T. Cao, X. Zhang, L. Kong, X. Liu, W. Shu, and M. Wu, "Traffic awarerouting in urban vehicular networks," in WCNC, pp. 2004-2009, 2013.

[2] P. Juang, H. Oki, Y. Wang, M. Martonosi, L. S. Peh, and D. Rubenstein, "Energy-efficient computing for wildlife tracking: Design tradeoffs andearly experiences with zebranet," SIGARCH Comput. Archit. News,vol. 30, no. 5, pp. 96-107, 2002. 
[3] Y. Zhang and J. Zhao, "Social network analysis on data diffusion indelay tolerant networks," in MobiHoc, pp. 345-346, 2009.

[4] A. Vahdat and D. Becker, "Epidemic routing for partially-connected adhoc networks," tech.rep., 2000.

[5] T. Spyropoulos, K. Psounis, and C. S. Raghavendra, "Spray andwait: An efficient routing scheme for intermittently connected mobilenetworks," in WDTN, pp. 252-259, 2005.

[6] A. Lindgren, A. Doria, and O. Schel'en, "Probabilistic routing in intermittentlyconnected networks," SIGMOBILE Mob.Comput.Commun.Rev., vol. 7, pp. 19-20, July 2003.

[7] J. Burgess, B. Gallagher, D. Jensen, and B. Levine, "Maxprop: Routingfor vehicle-based disruptiontolerant networks," in INFOCOM, pp. 1-11, April 2006.

[8] Q. Yuan, I. Cardei, and J. Wu, "An efficient prediction-based routingin disruption-tolerant networks," IEEE Trans. Parallel Distrib.Syst,vol. 23, no. 1, pp. 19-31, 2012.

[9] L. Vu, Q. Do, and K. Nahrstedt, "3r: Fine-grained encounter-basedrouting in delay tolerant networks," in WoWMoM, pp. 1-6, 2011.

[10] A. Lindgren, A. Doria, E. Davies, and S. Grasic, "Probabilistic routingprotocol for intermittently connected networks," Internet-Draft, 2012.

[11] J. Scott, R. Gass, J. Crowcroft, P. Hui, C. Diot, and A. Chaintreau,"CRAWDAD data set cambridge/haggle (v. 2006-01-31)."http://crawdad.org/cambridge/haggle/, 2006.

[12] N. Eagle and A. (Sandy) Pentland, "Reality mining: sensing complexsocial systems," Pers. Ubiq.Comput., vol. 10, no. 4, pp. 255-268, 2006.

[13] D. Kotz, T. Henderson, and I. Abyzov, "CRAWDAD data set dartmouth/campus (v. 2004-12-18)." http://crawdad.org/dartmouth/campus,2004.

\section{AUTHORS}

Luming Wan, is a Master candidate from Department of Computer Science, University of Otago, New Zealand. He obtained B.S. degree from University of Otago, New Zealand in 2012. His research interests include Delay Tolerant Network (DTN), Social Network, etc.

Feiyang Liu,is a PhD candidate from Department of Computer Science, University of Otago, New Zealand. He obtained B.S. and M.S. degrees of telecommunication engineering from Xidian University, China in 2009 and 2012, respectively. His research interests include Network on Chip (NoC), Optical Network on Chip (ONoC), Delay Tolerant Networks (DTN), etc.

Juan Zhang, received B.S degree in 2011 from Jiangsu University,China, and M.S. degree of control science and engineering in 2014 from Beihang University, China. Her research interests include distributed algorithms and protocols, wireless sensor networks, cyber-physical systems, and data communication, etc.

Haibo Zhang, received the MSc degree in Computer Science from Shandong Normal University, China in 2005, and the PhD degree in Computer Science from the University of Adelaide, Australia in 2009. From 2009 to 2010, he was a postdoctoral research associate at Automatic Control Laboratory, KTH, Sweden. Currently he is a lecturer at Computer Science department of University of Otago, New Zealand. His research interests include real-time industrial wireless communications, wireless sensor/ad hoc networks, delay-tolerant networks, green computing, distributed algorithms and protocol design. 